\title{
Performance of multilayer coated silicon pore optics
}

Ackermann, M. D.; Collon, M. J.; Cooper-Jensen, Carsten P.; Christensen, Finn Erland; Krumrey, M.; Cibik, L.; Marggraf, S.; Bavdaz, M.; Lumb, D.; Shortt, B.

\section{Published in:}

Proceedings of SPIE - The International Society for Optical Engineering

Link to article, DOI:

$10.1117 / 12.859823$

Publication date:

2010

Document Version

Publisher's PDF, also known as Version of record

Link back to DTU Orbit

Citation (APA):

Ackermann, M. D., Collon, M. J., Cooper-Jensen, C. P., Christensen, F. E., Krumrey, M., Cibik, L., Marggraf, S., Bavdaz, M., Lumb, D., \& Shortt, B. (2010). Performance of multilayer coated silicon pore optics. Proceedings of SPIE - The International Society for Optical Engineering, 7732(1), 77323U. https://doi.org/10.1117/12.859823

\section{General rights}

Copyright and moral rights for the publications made accessible in the public portal are retained by the authors and/or other copyright owners and it is a condition of accessing publications that users recognise and abide by the legal requirements associated with these rights.

- Users may download and print one copy of any publication from the public portal for the purpose of private study or research.

- You may not further distribute the material or use it for any profit-making activity or commercial gain

- You may freely distribute the URL identifying the publication in the public portal 


\title{
Performance of multilayer coated silicon pore optics
}

\author{
M. D. Ackermann ${ }^{\mathrm{a}}$, M. J. Collon ${ }^{\mathrm{a}}$, C. P. Jensen ${ }^{\mathrm{b}}$, F. E. Christensen ${ }^{\mathrm{b}}$, M. Krumrey ${ }^{\mathrm{c}}$, L. Cibik ${ }^{\mathrm{c}}$, \\ S. Marggraf $f^{\mathrm{c}}$, M. Bavdaz, D. Lumb ${ }^{\mathrm{d}}$, B. Shortt ${ }^{\mathrm{d}}$ \\ ${ }^{a}$ cosine Research BV, Niels Bohrweg 11, NL-233 CA Leiden, Netherlands \\ ${ }^{\mathrm{b}}$ DTU-space, Juliane Maries Vej 30, DK-2100 Copenhagen, Denmark \\ ${ }^{c}$ Physikalisch-Technische Bundesanstalt, Abbestr. 2-12, D-10587 Berlin, Germany \\ ${ }^{d}$ European Space Agency, ESTEC, Keplerlaan 1, PO Box 299, 2200 AG Noordwijk, Netherlands
}

\begin{abstract}
The requirements for the IXO (International X-ray Observatory) telescope are very challenging in respect of angular resolution and effective area. Within a clear aperture with $1.7 \mathrm{~m}>\mathrm{R}>0.25 \mathrm{~m}$ that is dictated by the spacecraft envelope, the optics technology must be developed to satisfy simultaneously requirements for effective area of $2.5 \mathrm{~m}^{2}$ at $1.25 \mathrm{keV}$, $0.65 \mathrm{~m}^{2}$ at $6 \mathrm{keV}$ and $150 \mathrm{~cm}^{2}$ at $30 \mathrm{keV}$. The reflectivity of the bare mirror substrate materials does not allow these requirements to be met. As such the IXO baseline design contains a coating layout that varies as a function of mirror radius and in accordance with the variation in grazing incidence angle. The higher energy photon response is enhanced through the use of depth-graded multilayer coatings on the inner radii mirror modules. In this paper we report on the first reflectivity measurements of wedged ribbed silicon pore optics mirror plates coated with a depth graded W/Si multilayer. The measurements demonstrate that the deposition and performance of the multilayer coatings is compatible with the SPO production process.
\end{abstract}

Keywords: silicon pore optics, multilayer coatings

\section{INTRODUCTION}

The International X-ray Observatory (IXO) is a collaborative venture between ESA, NASA and JAXA, which is under study for launch in 2020. IXO will have a launch mass of approximately $6500 \mathrm{~kg}$ and will be nearly 24 metres long in deployed configuration. The optics module contains the mirrors which, arranged in a double-conical approximation of the Wolter-I geometry, serve to focus the x-ray photons onto the focal plane instrumentation. Two technologies are under development for the IXO mirrors; silicon pore optics ${ }^{1}$ (SPO) and slumped glass optics ${ }^{2}$. The main scientific performance requirements and goals of the IXO telescope are summarised in Table 1.

\begin{tabular}{|l|l|}
\hline Parameter & Requirement / Goal \\
\hline Photon Energy Range & $0.3-40 \mathrm{keV}$ \\
\hline Mirror Effective Area $(1.25 \mathrm{keV})$ & $2.5 / 3 \mathrm{~m}^{2}$ \\
\hline Mirror Effective Area $(6 \mathrm{keV})$ & $0.65 \mathrm{~m}^{2} / 1 \mathrm{~m}^{2}$ \\
\hline Mirror Effective Area $(30 \mathrm{keV})$ & $150 \mathrm{~cm}^{2} / 350 \mathrm{~cm}^{2}$ \\
\hline Angular resolution (HEW) & $\begin{array}{l}\leq 5 \operatorname{arcsec} \mathrm{HEW}(0.1-7 \mathrm{keV}) \\
30 \operatorname{arcsec} \mathrm{HEW}(7-40 \mathrm{keV}) / 5 \operatorname{arcsec}\end{array}$ \\
\hline Field Of View & $18 \operatorname{arcmin}$ diameter \\
\hline
\end{tabular}

Table 1. IXO scientific performance requirements

Space Telescopes and Instrumentation 2010: Ultraviolet to Gamma Ray,

edited by Monique Arnaud, Stephen S. Murray, Tadayuki Takahashi, Proc. of SPIE Vol. 7732,

$77323 \mathrm{U} \cdot$ ? 2010 SPIE $\cdot$ CCC code: 0277-786X/10/\$18 · doi: 10.1117/12.859823

Proc. of SPIE Vol. 7732 77323U-1 
Due to the limited reflectivity of the mirror plate material $\left(\mathrm{Si}\right.$ or $\left.\mathrm{SiO}_{2}\right)$ at $\mathrm{X}$-ray wavelengths, it is recognised that specialised coatings will be required in order to meet the energy dependent effective area requirements of IXO.

For the SPO, the IXO mirror coating baseline is currently taken to be:

- all mirror modules from the innermost radius of $250 \mathrm{~mm}$ up to $\sim 450 \mathrm{~mm}$ shall be coated with a multilayer

- all mirror modules from $\sim 450 \mathrm{~mm}$ to the outermost radius shell $(\sim 1700 \mathrm{~mm})$ shall be coated with an Ir layer of thickness $10 \mathrm{~nm}$ and an B4C overcoat of constant thickness $8 \mathrm{~nm}$.

The application of an Ir $+\mathrm{C}$ coating and the subsequent bonding of ribbed silicon plates has recently been demonstrated ${ }^{3}$. Masked deposition was successfully used to ensure that the coating was only applied to the reflecting surfaces and thus allowing the $\mathrm{Si}-\mathrm{Si}$ bonding between adjacent mirror plates to be achieved. Measurements at the Four Crystal Monochromator (FCM) beamline (Physikalisch-Technische Bundesanstalt (PTB), BESSYII) verified the reflectivity enhancement as a function of photon energy.

It is well known that multilayer coatings can strongly enhance the reflectivity of mirror surfaces at hard X-ray wavelengths. These coating technologies have been applied to the problem of grazing incidence optics, where a wide range of solutions for graded layer thicknesses has been shown to offer a tunable energy range, especially for the energy band above the limits defined by the reflectivity of a single gold or iridium coating layer.

A previous paper ${ }^{4}$ described a set of measurements for low atomic number coatings on SPO including a W/SiC multilayer In this paper we report on the first reflectivity measurements of wedged ribbed SPO mirror plates coated with a depth graded $\mathrm{W} / \mathrm{Si}$ multilayer. The measurements demonstrate that the deposition and performance of the multilayer coatings is compatible with the SPO production process.

Reflectivity measurements at 20 and $30 \mathrm{keV}$ for uncoated and multilayer coated Si mirror plates have been performed at BAMline, a wavelength shifter (WLS) beamline at BESSYII jointly operated by the German Federal Institute for materials research and testing (BAM) and the Physikalisch-Technische Bundesanstalt (PTB). The results clearly demonstrate large reflectivity enhancement of the multilayer samples over the grazing incidence angle range relevant for IXO. An interlayer mixing of only $0.64 \mathrm{~nm}$ is derived from fitting to the reflectivity curves.

\section{MEASUREMENTS AND RESULTS}

\subsection{Samples and Coating Description}

The samples used during the reflectometry campaign were ribbed, wedged Si plates ${ }^{5}$, as used to construct X-ray Optical Units (XOU) within the SPO development program ${ }^{6}$. Several samples were uncoated with the reflective surface being the wedged $\mathrm{SiO}_{2}$ layer that results from the plate production process.

Several samples were coated with a depth-graded W/Si multilayer described by an $\mathrm{i}^{\text {th }}$ bilayer thickness distribution $\mathrm{d}(\mathrm{i})=$ $\mathrm{a} /(\mathrm{b}+\mathrm{i})^{\mathrm{c}}$. The multilayer is defined by the following parameters: $\mathrm{d}_{\min }=2.5 \mathrm{~nm}, \mathrm{~d}_{\max }=9.523 \mathrm{~nm}, \mathrm{~N}=291, \mathrm{c}=0.238, \gamma_{\min }=0.38$ and $\gamma_{\max }=0.8 . d_{\min }$ and $d_{\max }$ are the spacing thicknesses for respectively the first and last bilayers, $\mathrm{N}$ is the number of bilayers, $\mathrm{c}$ is the constant that determines how the multilayer develops, and $\gamma$ is the ratio of $\mathrm{W}$ in the layer. The constants $a$ and $b$ are determined from $d_{\min }$ and $d_{\max }$. The multilayer was deposited by DC magnetron sputtering at the DTU-Space institute in Denmark ${ }^{7}$. The multilayer was not specifically designed for the IXO telescope, but was rather a generic recipe that fits the incidence angle and energy range of IXO quite well. A future ESA technology development activity will look at IXO specific multilayer coating designs.

\subsection{Experimental Setup}

The BAMline delivers a collimated, monochromatic X-ray beam in the energy range of 20 up to $50 \mathrm{keV}$. The samples were measured in air, while being held down onto a flat vacuum chuck. The vacuum chuck was mounted on top of a set of high accuracy translation stages $( \pm 2 \mu \mathrm{m})$ and a set of goniometric cradles with an accuracy of \pm 2 " (see Figure 1). The complete assembly of translation and rotation stages was mounted on an optical table. The translation stages are used for 
alignment only; the cradles are used for both alignment and scanning of the samples. A set of slits delimits the incoming beam size to $0.1 \mathrm{~mm} \times 0.1 \mathrm{~mm}$ (vertical x horizontal).

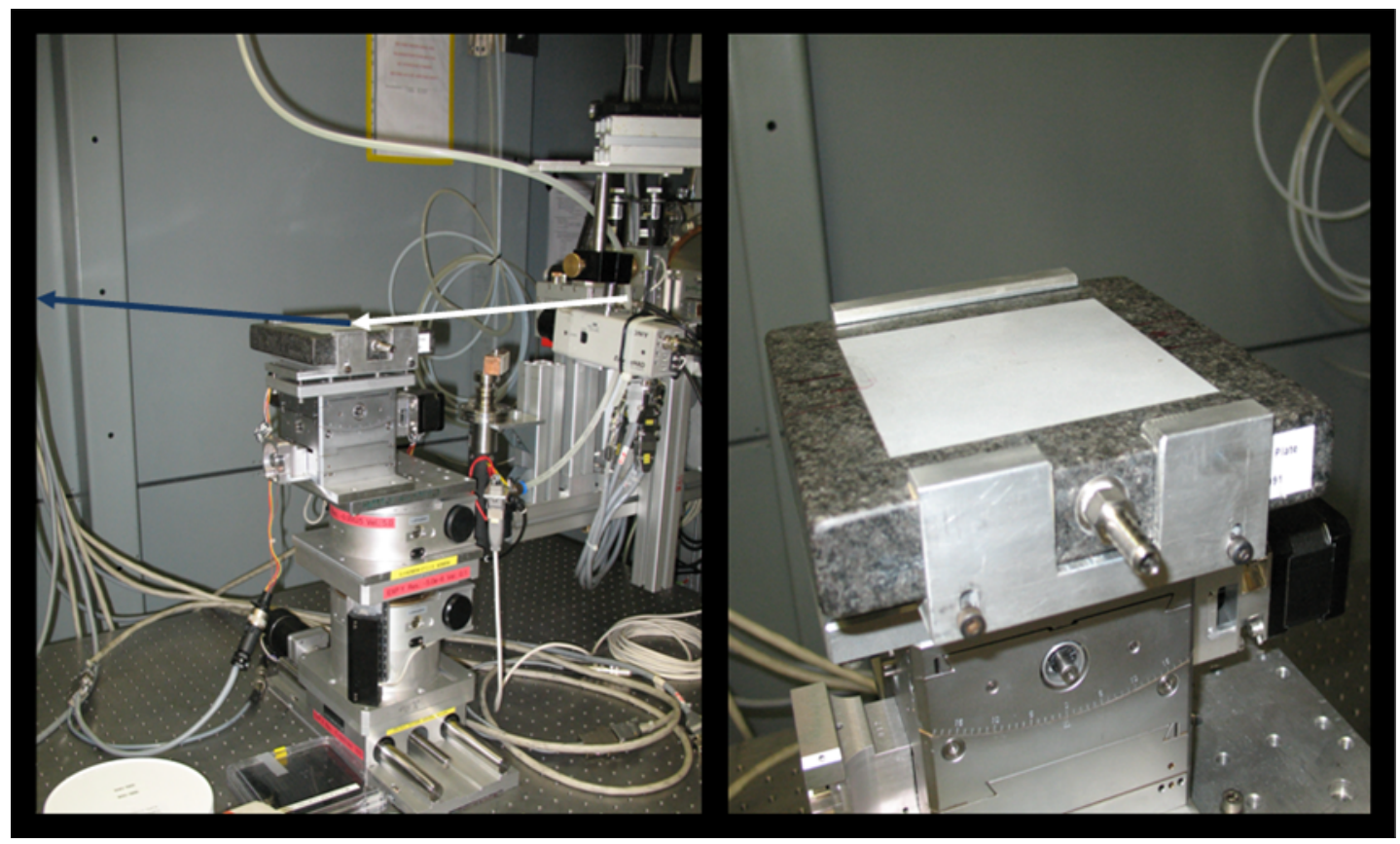

Figure 1: Setup at the BAM beamline of the synchrotron radiation facility BESSYII. Left: The complete setup with from bottom to top: optical table, rotation stage, translations stages, goniometer cradle stages and vacuum chuck. The incoming and reflected X-ray beams are represented by respectively a white and a black arrow. Right: a larger view of the vacuum chuck and goniometer cradles. The light gray centre area of the vacuum chuck is the porous material. On the side of the chuck is the connection for the vacuum pump.

Two detectors were used for these experiments. An X-ray diode, with a high dynamical range was used for large X-ray photon fluxes. When the detection limit of this diode was reached, the measurement was continued with a HPGe detector. This detector has a lower dynamical range, but a much better $\mathrm{S} / \mathrm{N}$ ratio. This detector is used when only a few photons are reflected from the sample (i.e. at high incidence angles). The HPGe detector has a background of 850 counts in a 5 second integration time. After suppression, the noise is reduced to \pm 30 counts / sec. Integration over more than 1 second further improves the $\mathrm{S} / \mathrm{N}$ ratio.

Both detectors are equipped with a slit which limits the acceptance of the detector in the vertical direction $(0.5 \mathrm{~mm})$, but not in the horizontal direction. This slit opening is much larger than the specular reflection of the X-ray beam from the sample, therefore integrating the whole reflected beam in the detector. The detector slit only limits (blocks) the acceptance of stray light and non-reflected X-rays straight from the direct beam at very low incidence angles.

\subsection{Scans}

The measurements reported here are derived from "theta-2theta" scans where the grazing incidence angle of the X-ray beam is scanned from 0 to $\mathrm{X}$ degrees, by rotating the sample. Simultaneously the detector is rotated around the same centre point, but by twice the angle of the sample to intercept the X-rays which are specularly reflected off the sample surface. The measurement of specularly reflected photon flux as a function of grazing incidence angle gives a reflectivity curve.

The scans have been corrected for several artifacts of the measurement in order to yield the absolute reflectivity of the samples. Corrections were applied for the variation in flux of the incoming beam and for the fact that at very low angles, the apparent height of the sample is smaller than the height of the incoming X-ray beam, and the reflected beam therefore shows a lower intensity. 


\section{RESULTS AND ANALYSIS}

\subsection{Measurement Results}

In Figure 2 we compare the reflectivity of a Si mirror plate without multilayer coating and one with the depth graded $\mathrm{W} / \mathrm{Si}$ multilayer at a photon energy of $20 \mathrm{keV}$. It is obvious that the addition of a multilayer coating greatly enhances the reflectivity of the mirror plates at high photon energies. For example, the innermost radius XOU's of IXO ( $20 \mathrm{~m}$ focal length, $0.25 \mathrm{~m}$ radius) have an incidence angle of 0.18 degrees. From Figure 2 we can see that the addition of the multilayer coating has increased the reflectivity at 0.18 degrees by a factor of about 200 .

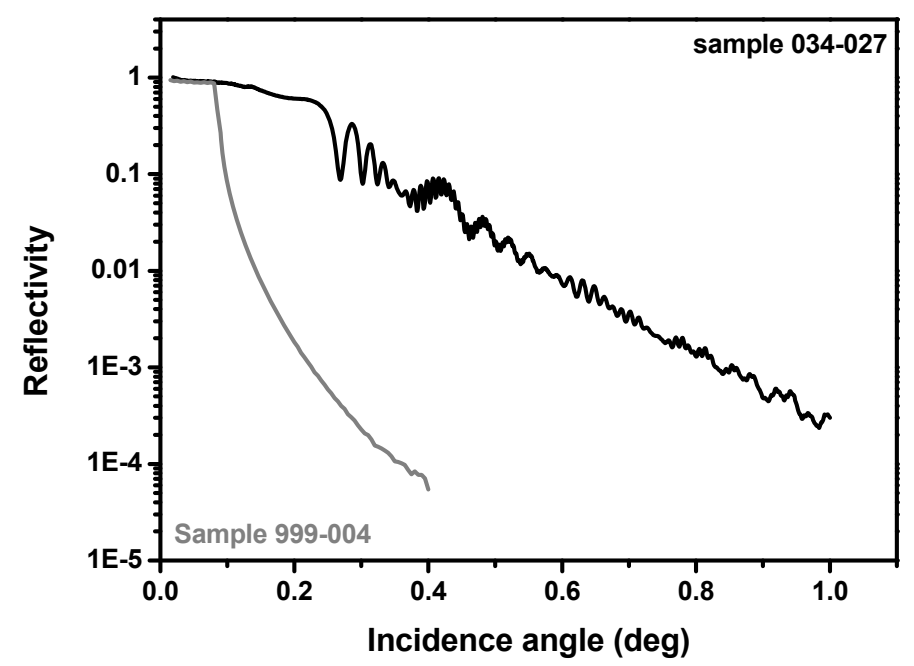

Figure 2: Reflectivity as a function of incidence angle at $20 \mathrm{keV}$ for a depth graded W/Si multilayer coated mirror plate (black line) and a bare, wedged Si mirror plate (grey).

In Figure 3 we present the reflectivity of the depth graded W/Si multilayer at photon energies of 20 and $30 \mathrm{keV}$. As expected, the reflectivity at $30 \mathrm{keV}$ is lower than at $20 \mathrm{keV}$ over almost the complete incidence angle range.

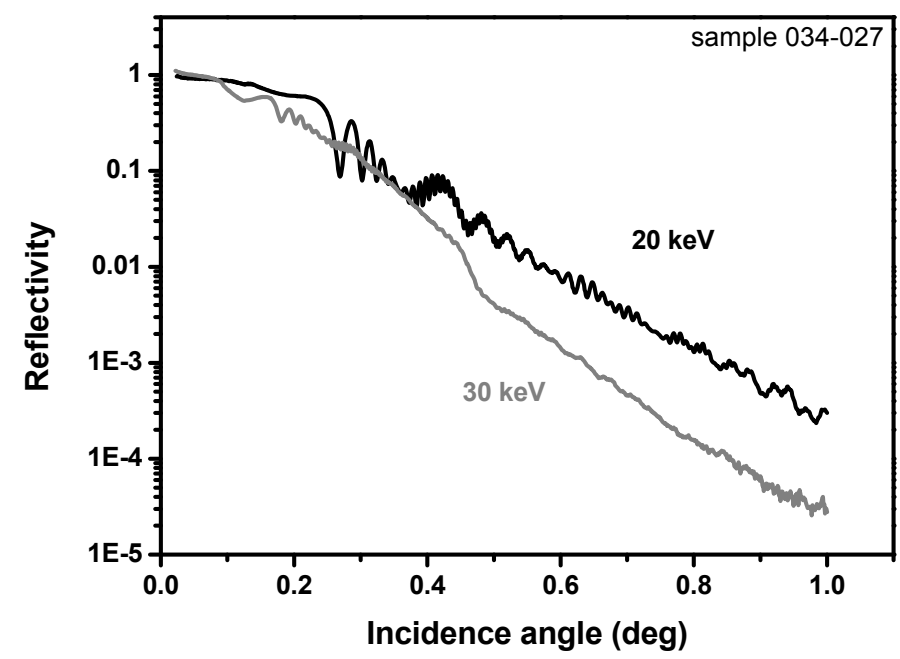

Figure 3: Reflectivity as a function of incidence angle at 20 and $30 \mathrm{keV}$ for a wedged Si mirror plate with a W/Si multilayer coating. 


\subsection{Analysis}

It is well known that pure $\mathrm{Si}$ reacts with oxygen in the air and forms a thin layer of (amorphous) $\mathrm{SiO}_{2}$, also known as 'native oxide'. In the case of the wedged mirror samples presented here, a thick (1 micron) layer of $\mathrm{SiO} 2$ has already been formed by thermal oxidation during the mirror plate production process. However, this oxide layer has been in contact with both air and etching chemicals (diluted HF). Both the contact with air and/or the contact with the HF could have caused the most outer layer of the oxide to adopt a slightly different density than the thick thermally formed oxide. In figure 4 we show both the reflectivity data from the wedged oxide layer, and from a fit to this data. From the fit to the reflectivity data, we conclude that a layer of $1.25 \mathrm{~nm}$ shows a different density than the 'bulk' $\mathrm{SiO}_{2}$ below. The density of this top $1.25 \mathrm{~nm}$ is approximately $1.28 \mathrm{~g} / \mathrm{cm}^{3}$ which is significantly less than the $2.3 \mathrm{~g} / \mathrm{cm}^{3}$ of the bulk $\mathrm{SiO}_{2}$. As these measurements were performed in air and without a prior cleaning procedure for the Si mirror plates, adsorbed layers of water or carbonaceous species can slightly influence the reflectivity and therefore the fitted thickness and density of the top $\mathrm{SiO}_{2}$ film. A roughness value of $0.4 \mathrm{~nm}$ was used in the fitting.

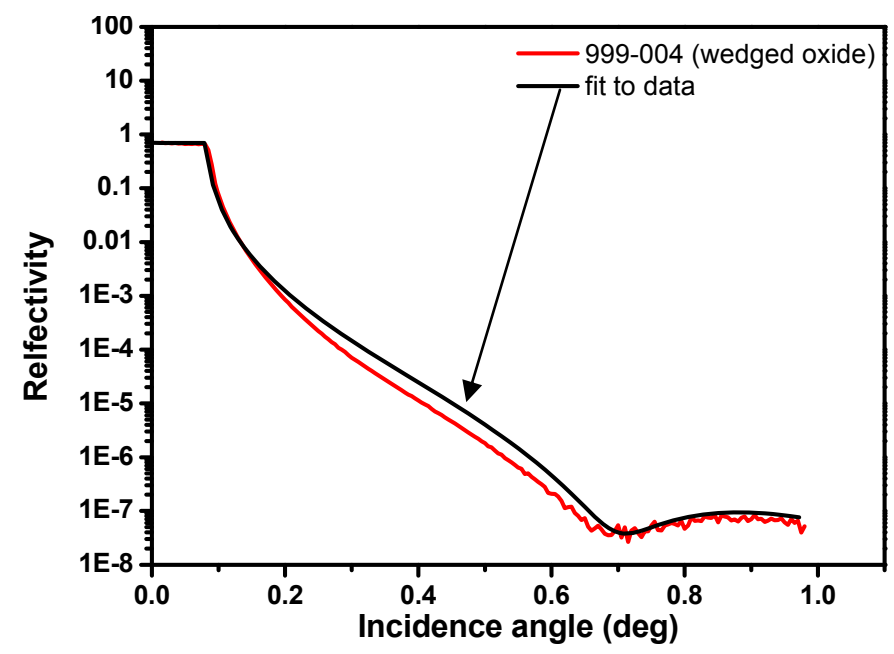

Figure 4: Measured (red) and fitted reflectivity (black) curves of the uncoated wedged silicon mirror plate at $20 \mathrm{keV}$ photon energy.

In Figure 5 we show a fit to the reflectivity curve of the depth graded multilayer. We have simulated the reflectivity of a depth graded multilayer with the parameters as described in section 2.1 of this document. The only parameters that have been allowed to relax are the $\mathrm{W}$ and Si layer thicknesses, and the interlayer mixing or diffuseness of the interface between the Si and W. Especially in the range from 0 to 0.5 degrees, the fit matches the data very well. The best fit is achieved with an interlayer mixing of $0.64 \mathrm{~nm}$ and thicknesses for both the $\mathrm{W}$ and $\mathrm{Si}$ layer which are slightly higher than the nominal values stated in section 2.1 of this document. The substrate surface roughness cannot be fitted from this data, but must be better than the interlayer mixing of $0.64 \mathrm{~nm}$. 


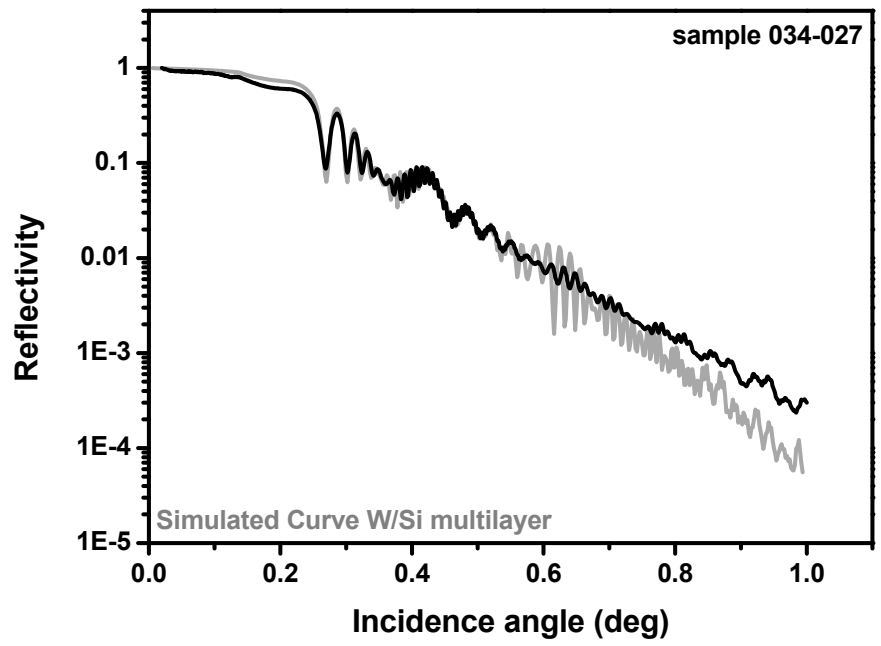

Figure 5: Fit (grey) to the measured reflectivity (black) curve of the multilayer coating. The fitting parameters have been optimized for the data measured between 0 and 0.5 degree incidence angle as this is the area of interest for calculating an effective area for IXO. Beyond 0.5 degrees, the reflectivity drops below $1 \%$ (single reflection), and contributes very little to the effective area.

Using the multilayer recipe described in section 2.1, we have also simulated the expected reflectivity at $30 \mathrm{keV}$ using the Windt IMD code 8 . This is shown in Figure 6 . Note that this is a simulation and not a fit; no parameters have been optimized and the results highlight the consistency of the multilayer coatings.

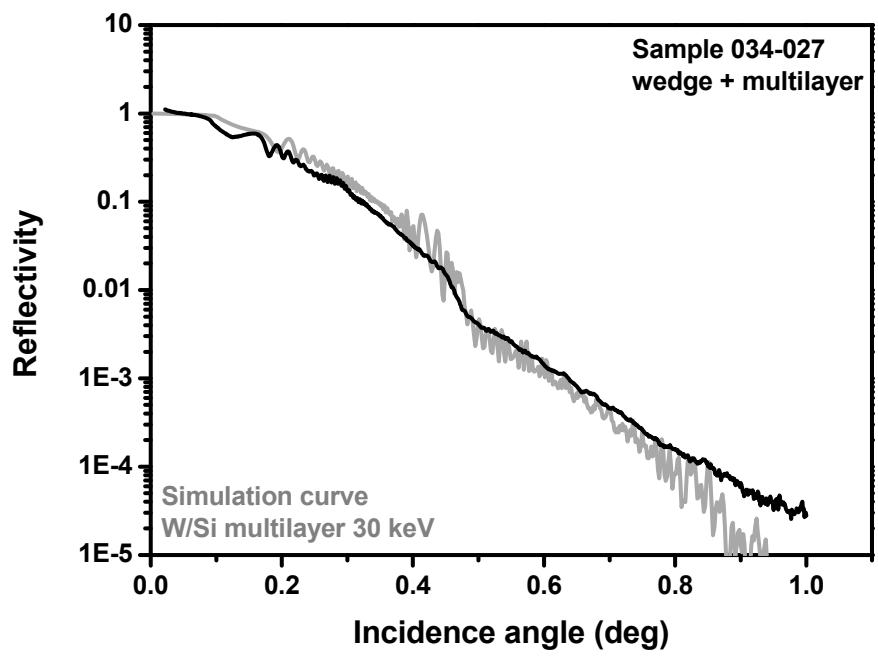

Figure 6: Simulation (grey) and measured reflectivity (black) curves of the depth graded W/Si multilayer coating at $30 \mathrm{keV}$ photon energy. 


\section{CONCLUSIONS}

Within the context of the wider IXO Silicon Pore Optics technology development program, multilayer coatings have successfully been deposited and characterized on a set of ribbed wedged $\mathrm{Si}$ mirror plates for the first time. This demonstrates the compatibility of the coating and deposition process with the SPO production process.

$\mathrm{X}$-ray reflectivity measurements at 20 and $30 \mathrm{keV}$ show that the multilayer produces the significant reflectivity enhancement which is critical to meeting the effective area requirements. A fit to the reflectivity curve at $20 \mathrm{keV}$ shows that the actual properties of the multilayer are very close to the nominal parameters set by DTU Space in terms of layer thickness for the $\mathrm{Si}$ and $\mathrm{W}$. The interlayer mixing is in the order of $0.6 \mathrm{~nm}$. Simulations of the reflectivity of this multilayer at $30 \mathrm{keV}$ are very close to the measured data.

The multilayer coating applied to the Si plates was not specifically designed for IXO. It therefore does not represent the final performance that can be achieved with IXO at these photon energies. It however gives a good estimate for what is achievable with multilayer coated Si mirrors in general. By tuning the parameters of the multilayer specifically for IXO, it is expected that the effective area requirements will be met. This will be the subject of a future technology development activity.

\section{REFERENCES}

[1] Wallace, K., Bavdaz, M., Gondoin, P., Collon, M.J., Günther, R., Ackermann, M., Beijersbergen, M.W., Olde Riekerink, M, Blom, M., Lansdorp, de Vreede, L., "Silicon Pore Optics Development," Proc. SPIE 7437, 74370T (2009).

[2] Pareschi, G., Ghigo, M., Basso, S., Citterio, O., Canestrari, R., Dell’Orto, E., Conconi, P., Parodi, G., Proserpio, L., "Design and Development of the IXO Mirrors by Innovative Slumping Glass Technologies," American Astronomical Society, AAS Meeting \#213, \#457.08, Bulletin of the American Astronomical Society 41 (2009).

[3] Jensen, C.P., Ackermann, M., Christensen, F.E., Collon, M.J., Krumrey, M., "Coating of silicon pore optics", Proc. SPIE 7437, 743713 (2009).

[4] Lumb, D.H., Jensen, C.P., Krumrey, M., Cibik, L., Christensen, F., Collon, M., Bavdaz, M., "Low atomic number coating for XEUS silicon pore optics," Proc. SPIE 7011, 70111D (2008).

[5] Olde Riekerink, M.B., Lansdorp, B., de Vreede, L.J., Blom, M.T., van 't Oever, R., Ackermann, M.D., Collon, M.J., Wallace, K., Bavdaz, M., "Production of Silicon Mirror Plates," Proc. SPIE 7437, 74370U (2009).

[6] Collon, M.J., Günther, R., Ackermann, M., Partapsing, R., Kelly, C., Beijersbergen, M.W., Bavdaz, M., Wallace, K., Olde Riekerink, M., Müller, P., Krumrey, M., "Stacking of Silicon Pore Optics for IXO," Proc. SPIE 7437, 74371A (2009).

[7] Jensen, C.P., Christensen, F.E., Chen, H.C., Smitt, E.B.W., Ziegler, E., "Multilayer coating facility for the HEFT hard x-ray telescope,"Proc. SPIE 4496, 104 (2002).

[8] Windt, D.L., "IMD - software for modeling the optical properties of multilayer films," Computers in Physics $12,360-270$ (1998). 